\title{
Multileaf Spring Model and Its Behaviour in a Tandem Bogie Layout
}

\author{
Martin Maloch, Stefan Cornak \\ University of Defence in Brno \\ Kounicova 65, Brno, Czech Republic \\ martin.maloch@unob.cz; stefan.cornak@unob.cz
}

\begin{abstract}
The utilization of independent suspension is rising. For a very specific conditions, the dependent suspension types are still preferred, mainly in case of heavier trucks. Ordinary leaf spring material-steel is being replaced by a variety of composite materials. For the dynamic simulation of the vehicle, the greater importance than a material type, lies in the layout of the suspension. The tandem bogie layout of the multileaf spring suspension has many differences in its behaviour in comparison with ordinary layout- set of two separated multileaf springs allocated to one axle. In the first part of the paper, these differences are listed and explained- mainly the ability of load sharing between the axles. The second part deals with specific aspects of the FEM model, along with the necessary analytical background. The third part consists of various simulation cases with different initial conditions- different constrains, pretension and spring as tyre stiffness. In the last part, the effect of the aforementioned conditions are evaluated and commented. The variations of the hysteresis loops, in force-displacements characteristics, along with derived linear stiffness's and its behaviour are recommended to be understood for additional application. Therefore, the conclusions are drawn for further utilization of gathered data for the full vehicle simulation.
\end{abstract}

Keywords: Multileaf spring, Tandem bogie, FEM, Hysteresis loop.

\section{Introduction}

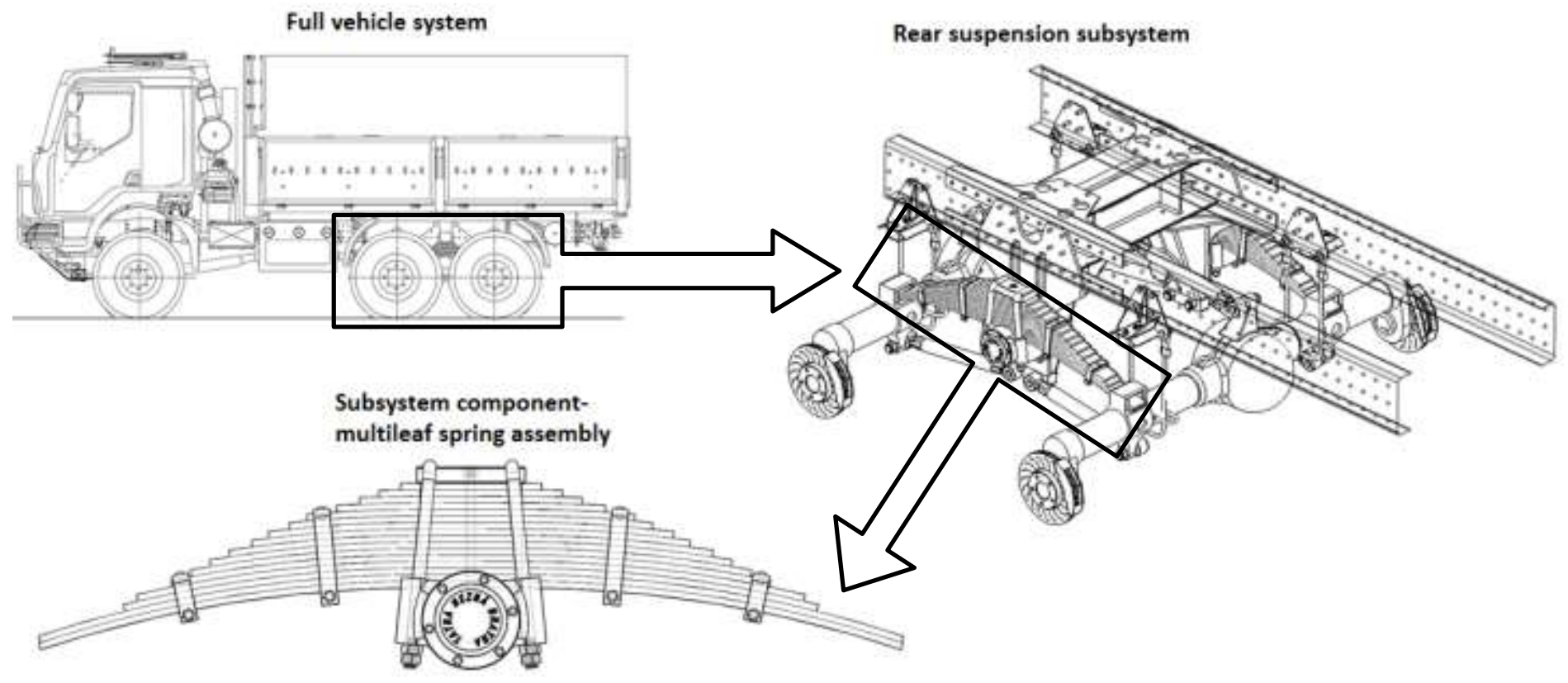

Fig. 1: Illustration of the topic of this paper in terms of full vehicle system.

The study of dynamic behaviour of vehicle can be conducted with proper simulation methods along with the model of full vehicle system. Unfortunately, the full vehicle system (fvs) is so complex, that firstly the vehicle system needs to be broken down into a several subsystems-e.g. front and rear suspensions, drivetrain, chassis, cabin etc. In some cases, even the subsystems are too complex. Since the successful evaluation of simulation results is conditioned by the full comprehension 
of the subject, the subsystems are further divided into subsystem components or assemblies. This approach can be observed from Fig. 1. Even the simplest part from the aforementioned figure required previous study of the multileaf spring behaviour and its characteristics. In [1], the basic approach to the modelling of prestressed multileaf spring is described. Presented in Fig. 2 are the components of the rear suspension subsystem. The difference in locations of the centre of the axle and wheel hub is caused by the additional gearing reduction.

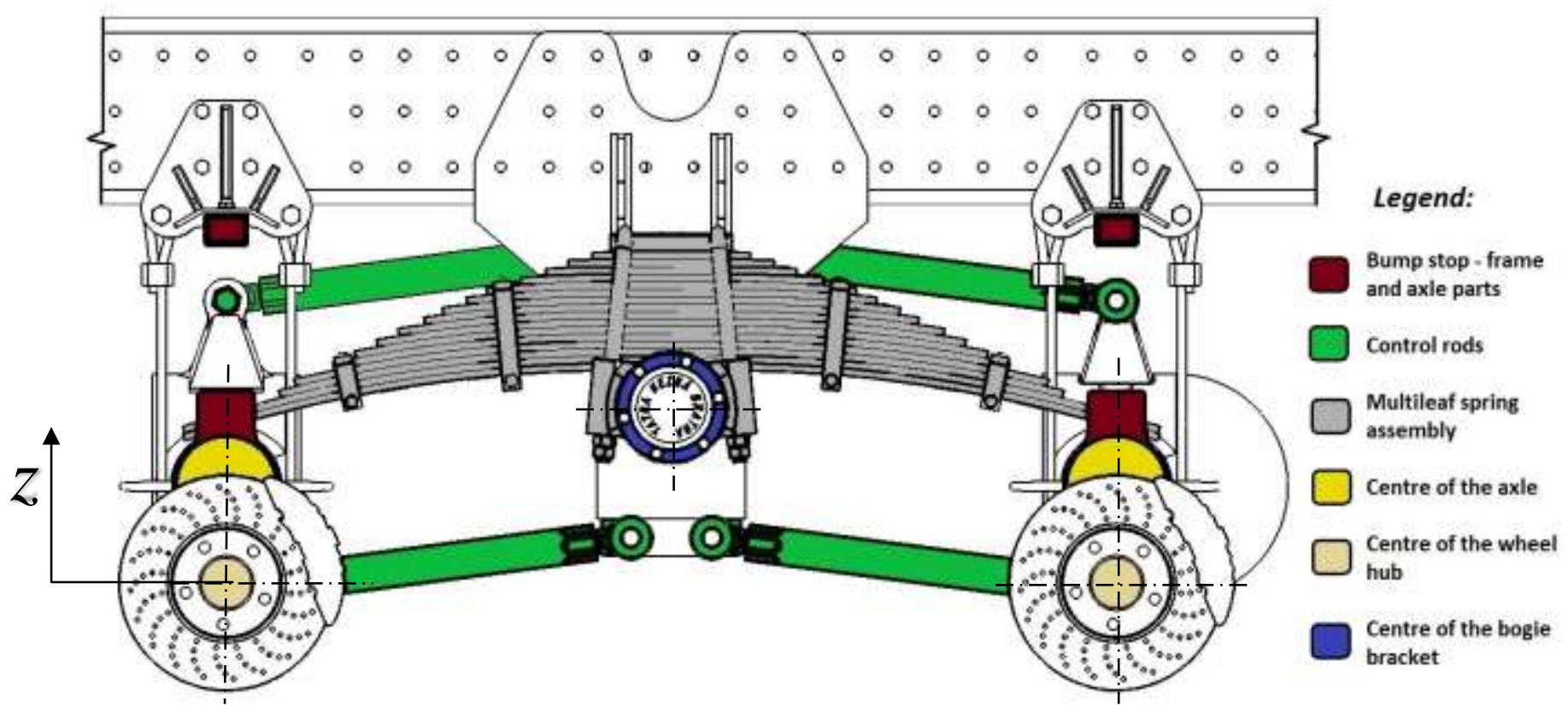

Fig. 2: Layout and components of tandem bogie suspension with multileaf spring.

The main advantage of the tandem bogie layout can be employed in vehicles which have both axles driven. If the wheel is excited due to bump or any obstacle, its position in $\mathrm{Z}$ axis will change upward. Since the leaf spring is an elastic element, it will deflect. Also, the whole multileaf spring assembly pivots around the centre of the bogie bracket. Created leverage should press the other wheel in the opposite direction, therefore significantly increasing its ability of transferring the driving forces from wheel to ground. This process is called load sharing. In muddy terrain, if the obstacle needs to be overcome, this leverage can improve the chances of the vehicle of not being stuck- higher transferable driving forces can significantly improve the traction and mobility of the vehicle in difficult terrain.

Obviously, the behaviour of the multileaf spring assembly will be the governing input in dynamic simulation of the fvs. Many publications, as stated in [1] are focusing on the leaf spring simulations and modelling, but fewer are focused on this particular layout. In [2], the authors are dealing with very similar problematic, although with simplification that would in this case devalue the results of the simulation- e.g. the absence of clamps, the uniting of the inactive length. The assembly utilizes only 4 leaves, whereas in this particular system there are 16 leaves. The differences are caused by the different computation techniques- the model from the aforementioned article is for Multi Body System (MBS), whereas in this article the FEM method is applied. Both approaches have specific advantages and disadvantages which should be carefully evaluated before the simulation.

The most suitable solution would be implementation of the behaviour studied in FEM model to MBS model, although this task can be very challenging and time consuming. The behaviour of leaf spring assembly is a very complex topic due to a possible variation of the inputs. The trend in case of leaf spring problematic is the comparison of the stresses, fatigue and weight reduction between various materials- mainly steel and composites. For behavioural study, 
the biggest challenges lies within the variations of the coefficient of friction (COF). For assembly with 16 leaves and a high amount of contact surfaces, the effect of COF is significant. Its value is affected by many factors, e.g. long term weather conditions- rust, frequency of the driving with maximal load, the amount of dirt, dust and debris between the leaves etc. Therefore, achieving the desired correlation between the fvs model and experimental study can prove to be very challenging.

\section{Specific aspects of suggested FEM model}

The best option for the dynamic simulation is the MBS approach. While reproducing of the front suspension from the fvs can be relatively easily done, the rear suspension subsystem requires much more attention. In [3], the MBS simulation of the ordinary suspension was carried out, along with its verification by state space model. The elastic and damping elements were linear, therefore simple to implement to the computations. For this paper, the FEM software Ansys was utilized.

Firstly, the geometry of all necessary parts was modelled in CAD software. Afterwards it was exported as .step file and imported to Ansys. All of the components are presented in Fig. 3. In this article, three possible components can be prestressed.

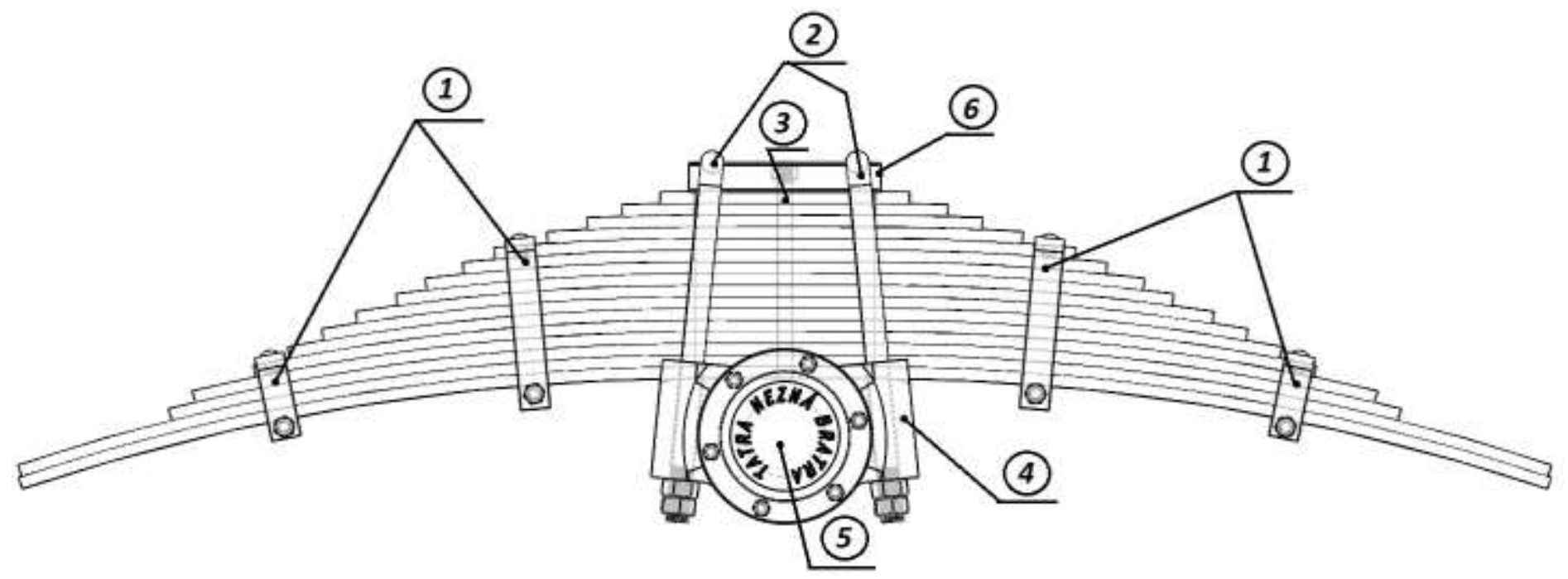

Fig. 3: Multileaf spring assembly: the stack of leaves.(1)- long and short clamps, (2)- set of U-bolts,(3)- central bolt,(4)- bogie bracket, (5)- cover of a pin which connects the assembly with the chassis of the vehicle,(6)- saddle for the U-bolts.

In Ansys, the bolt pretension tool [4] can be fully utilized for this purpose. The pretension can be defined in 3 possible ways- by the force, adjustment and increment. For this process, one load step during simulation is required. After reaching the required value of pretension, the bolt pretension tool is set to "lock", so the other load steps might begin. According to [5], the pretension force value was calculated from equations (1) and (2). Its verification within the simulation model was made by comparison of the stress values- calculated in equation (3) and simulated ones.

$$
\begin{gathered}
F_{P t}=\frac{T}{S_{c} \cdot d_{r}} \\
S_{c}=\frac{d_{m}}{d_{r}} \cdot\left[\frac{\tan \lambda+f \cdot \sec (\alpha / 2)}{1-f \cdot(\tan \lambda) \cdot \sec (\alpha / 2)}\right]+0.625 \cdot f_{\circ} \\
\sigma_{P t}=\frac{F_{P t}}{A_{t}}
\end{gathered}
$$


Where $F_{P t}$ is the pretension force, $T$ is the torque value, $S_{c}$ is the stiffness constant, $d_{r}$ is minor diameter of the bolt, $d_{m}$ is the mean diameter of the bolt, $\lambda$ is the lead angle of the thread, $\alpha$ is the thread angle and according to [5], the $f_{\mathrm{o}}$ and $f$ are equal and represents the coefficient of friction within the threads. The $\sigma_{P t}$ is the preload stress and $A_{t}$ is the tensie-stress area.

The preloaded components are the central bolt, set of U-bolts. As can be seen in Fig. 3 the clamps- position 1 , are made of bent rectangular profile, although some simulation cases will be mentioned, where these clamps are replaced with the ones with circular profile. This replacement will enable the further utilization of the pretension tool in Ansys, which is bound to the circular surface. Pretension of the noncircular surfaces is possible, but it is more complicated and time consuming.

The main problem of the multileaf spring simulation lies in numerous contact surfaces. The main goal was to achieve as realistic results as possible, therefore in terms of contacts- not a single pair was omitted. All of the contact settings were primarily set due to knowledge gained from [6], followed by the method of trial and error. The majority of 95 contacts in simulation was frictional or frictionless- if the friction effect was evaluated as omittable in terms of global behaviour of the multileaf spring- e.g. the contact between the sidewall of the leaf and a clamp. For the contact detection, the Augmented Lagrange formulation was utilized. Another important element was the size of the pinball region- this provides the computational efficiency in the contact calculations by differentiating between the "near" and "far", in terms of searching for elements which can possibly contact each other in given contact region.

If the problems with a convergence occurred, the Newton-Rhapson residuals have been investigated and further arrangements were implemented within the problematic region. Another important feature is the "Contact Tool- status". With this tool, a chosen contact pairs can be shown and observed. The observation is relatively simple, as illustrated in Fig. 4- by comparison of the colours of contact pairs with legend provided, the conclusions can be drawn and implemented to model.
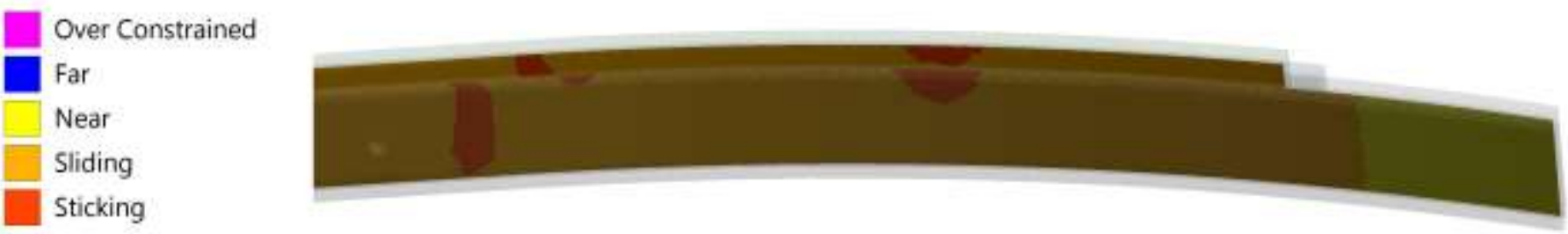

Fig. 4: Contact Tool- status: illustration of bottom 3 leaves of the assembly.

The properties of the mesh have to be considered carefully due to huge impact on the simulation results along with computational time. The "Body Sizing" function was utilized for the clamps and U-bolts and the "Contact Sizing" for the contact pairs between the individual leaves.

\section{Conducted simulation cases}

As mentioned before, the simulations were conducted with a several different initial conditions and constrains. In Fig. 5 is presented the model of the multileaf spring, where all of the mentioned initial conditions and constrains are illustrated. The Global Coordinate System, or G. C. S. is valid for the whole assembly and the most important point is represented by Local Coordinate System or L. C. S. This point is the location of constrain of the whole assembly for the first few simulationsbasically it represents the middle of the hole of bogie bracket. Firstly, the assembly is fixed by fixed support- every motion is restricted. The only force applied is the $\mathrm{F}_{\mathrm{i}}$ - the input force.

Secondly, the fixed support is replaced by the cylindrical support- the only degree of freedom for the assembly is the rotation about $\mathrm{z}$ axis (indicated by green arrow). The end of the assembly is supported by the frictionless support, afterwards the assembly is loaded by $F_{i}$. For even more realistic measurements, the frictionless support is replaced by the spring. The properties of the spring represents the elastic behaviour of the wheel- the position, length and stiffness are the same as in the simulation conducted in [3]. 
Thirdly, the A.C- Additional Component is introduced as the auxiliary component. Its sort of a pin is inserted in the bogie bracket's hole and the frictional contact pair is created. Both components have constrained the movement in the $\mathrm{Z}$ axis $\mathrm{Z}$ axis direction. The only direction which has the free movement is in $\mathrm{Y}$ axis for the A.C. The assembly is preloaded with with the half of the maximal weight of the vehicle for the rear suspension, represented by $F_{v}$ in the figure. The second spring spring is introduced for the other side of the assembly, then the assembly is loaded with input force. The best solution would would be the compression only spring. This problem can be solved by introducing another set of auxiliary components and constrains. Unfortunately, the aforementioned solution is beyond the scope of presented paper.

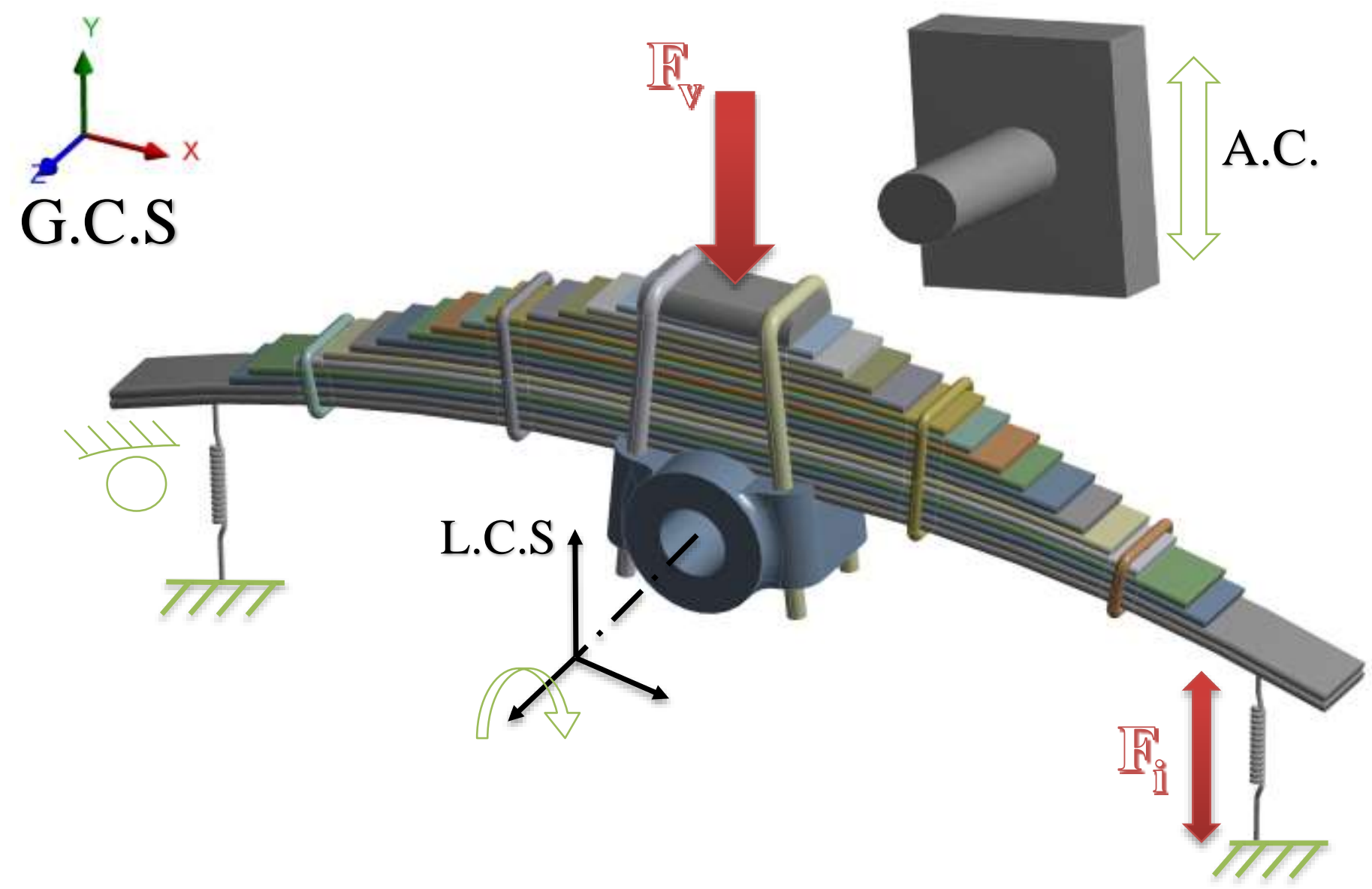

Fig. 5: Illustration of various constrains and initial conditions applied to the simulation model.

\section{The results of the simulations}

Individual simulations are compared in terms of multileaf spring behaviour- governed by the force-displacement characteristics. Due to friction between the leaves, these characteristics should evince the hysteretic behaviour, which is supposed to rise with the growth of COF. According to [7], up to three stiffness regimes should be observed. Basically, two values of stiffness represents the loading and unloading stiffness of the layered beam. After the reaching of the ultimate frictional yield force, the third stiffness regime for the solid beam can be seen. Then, the force is decreasing up to the frictional yield force, where the stiffness is incrementally changing to the unloading one of the layered beam. As can be seen in Fig. 5 , the direction of input force is identical to the positive direction of the global $\mathrm{Y}$ axis. Therefore, during the loading cycle the input force have a rising positive value. 


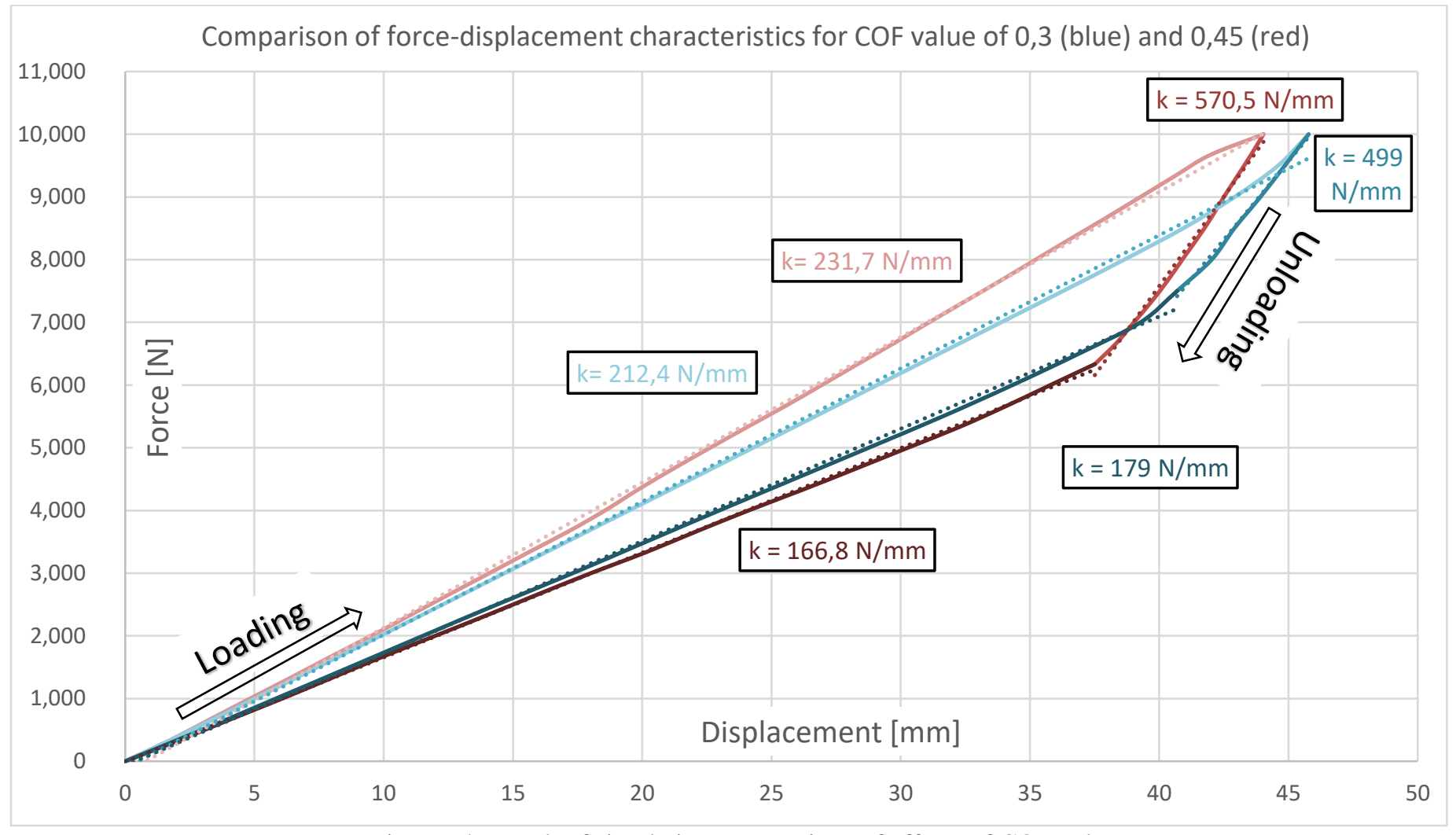

Fig. 6: The result of simulation- comparison of effects of COF values.

Presented in the Fig. 6 above, is the force-displacement characteristic of the two primal simulations, fixed support constrain in the bogie bracket with the input force loading the plain end of the leaf spring. The clamps are not prestressed. The values of the COF are relatively high due to the illustrative purposes. All of the three mentioned stiffness regimes can be observed, the values of stiffness are coloured correspondingly with the curves. Also, the effect of the higher COF value is clearly visible. Firstly, the value of the maximal displacement is lower than in case of lesser COF value. With the same input force, the difference between the displacements is mainly caused by the amount of energy required to overcome the overall resistance of the assembly. This resistance is rising with the rise of COF value, so the leaf spring with higher COF value have a stiffer behaviour. Also, the damping is higher due to significantly higher amount of dissipated energy. The damping caused by the energy dissipation should be a subject of further research, so the damping coefficient with its properties can be set. Unfortunately, such a research is beyond the scope of presented paper.

The effect of the prestressed clamps can be seen in the Fig. 7. Various values of the prestress level are simulated with the cylindrical support in the bogie bracket, the spring on the other end of the assembly and the input force located just like in the previous simulation. The stiffness of the result should be halved in comparison with previous simulation. The value of COF was chosen to be 0,25 . The solid beam stiffness value was linearized and its values are presented in the figure below. As expected, with rising pretension value, the overall stiffness of the assembly is rising too. The difference in displacement is $8 \mathrm{~mm}$ between the low and high pretension values. The rising pretension is also prolonging the solid beam behaviour, as can be seen from the coloured lines in the figure. The loading stiffness's, were linearized and their values are presented in the top right corner of the Fig. 7. As expected, the values $\mathrm{k}_{\mathrm{HP}}, \mathrm{k}_{\mathrm{MP}}, \mathrm{k}_{\mathrm{LP}}$ (high, medium and low values of pretension) for loading part of the cycle are approximately halved, compared to the value of the stiffness in the loading cycle from the Fig. 6. The difference in the rest of the cycle is caused by the COF value and spring stiffness along with the inactive length of the leaves in the area around the central bolt. 


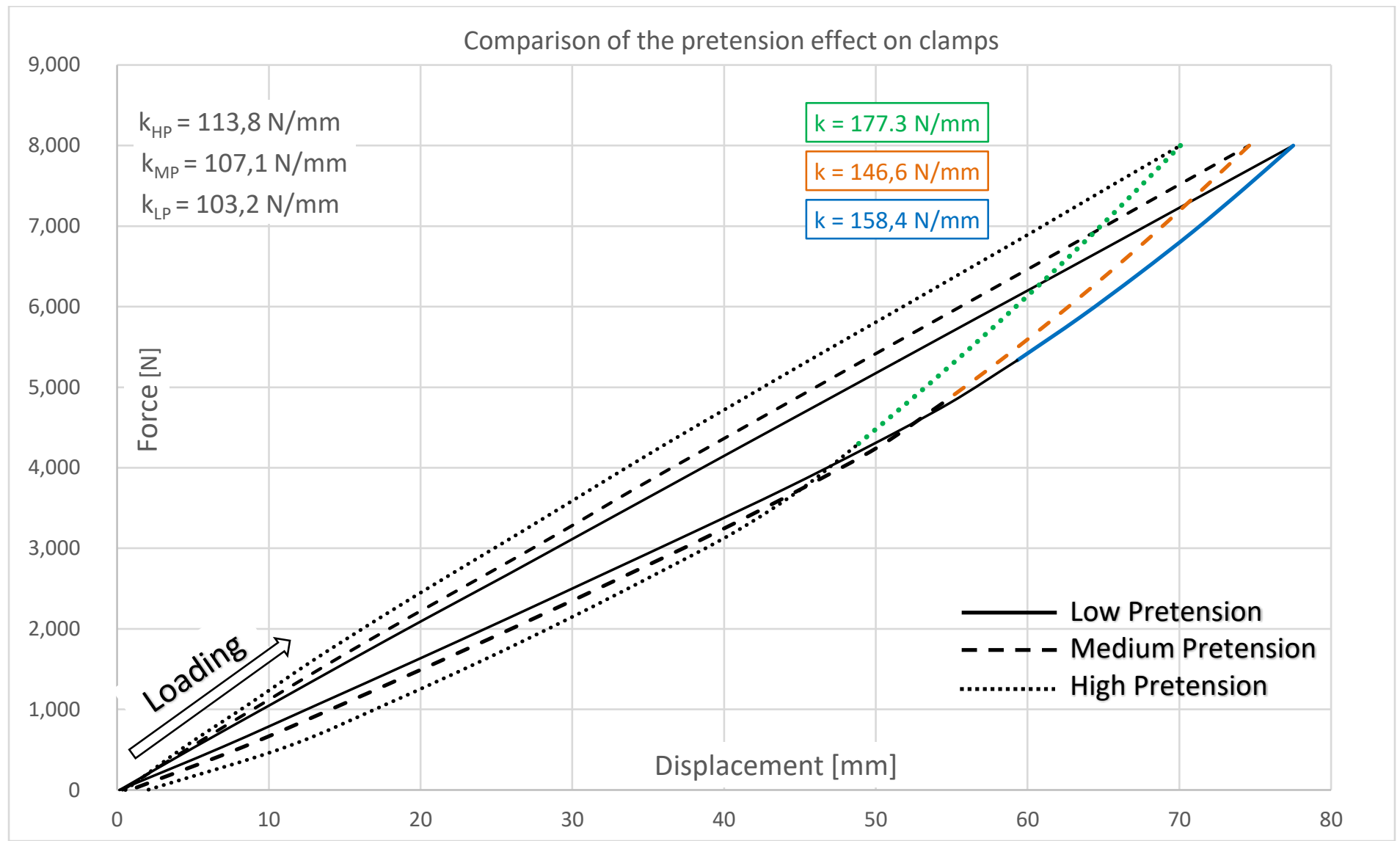

Fig. 7: The result of simulation- pretension effect on clamps.

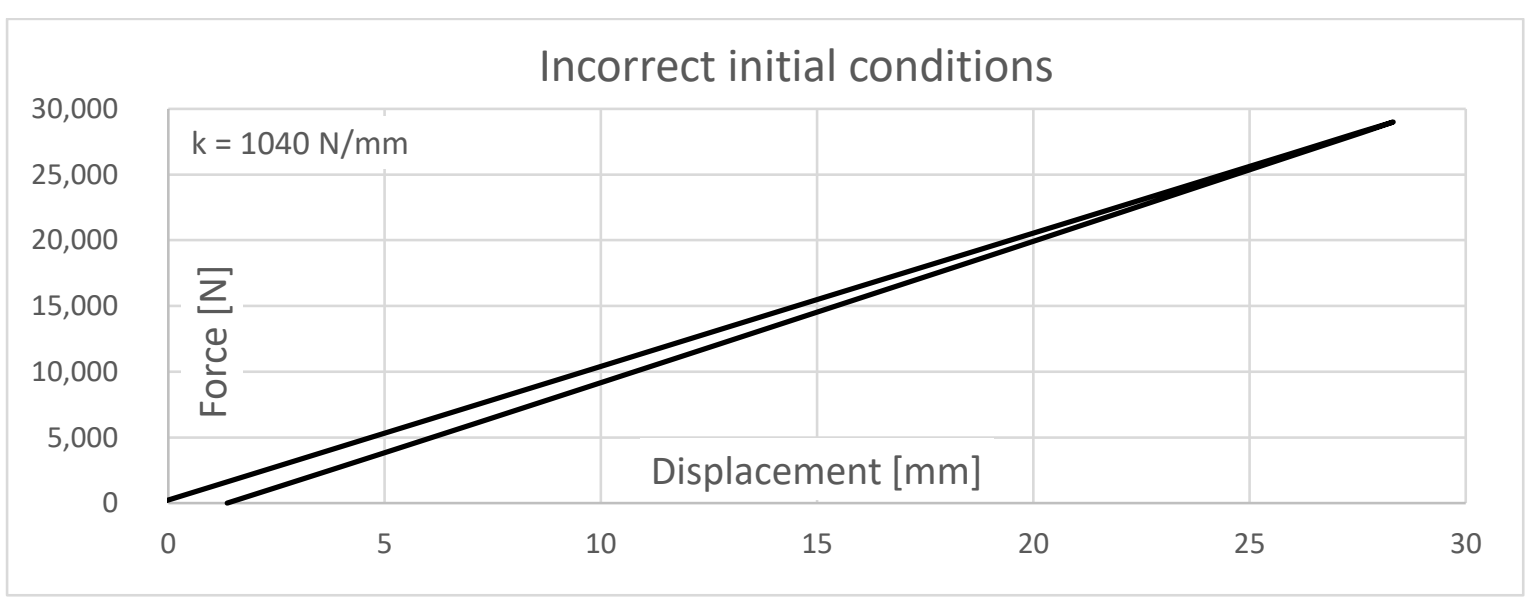

Fig. 8: The result of simulation- incorrect initial conditions.

As mentioned before, the simulation with the most complex initial conditions- springs on both ends of the assembly, additional contact pair with the A.C. and added preload (half of the weight of the vehicle), was expected to deliver the 
incorrect results. The linearized stiffness if highly affected by the stiffness of the spring (tyre replacement), which is significantly higher. The rest of the reasons were described in the previous chapter.

\section{Discussion and Conclusion}

The study of the multileaf spring behaviour, in terms of tandem bogie layout has, been suggested. The aims of the were fulfilled and the force-displacement characteristic has been created. According to [7], the characteristic is evincing the expected behaviour with three different stiffness regimes. Furthermore, the effect of the prestress within the clamps was observed.

The biggest simplifications utilized during the simulations were related to the value of COF. Firstly, only one constant value was used. The proper way would be with static and dynamic value, so the beginning of the loading curve would have different shape. Secondly, the value of COF would be different for different pairs of leaves- if the vehicle was operated with small additional weight (cargo, crew), after the fully loaded operation the upper leaves might have a higher value of COF due to corrosion between the less used leaves. On the other hand, in between the bottom leaves the COF value might be higher due to dust and debris from the ground. Also, the distance from the input force to L.C.S was set to a constant number - but according to Fig. 2, the motion of the axle and therefore the input force is governed by control rods and the aforementioned distance should have been slightly varying during the simulation.

The simulation model might be utilized as the replacement for the experimental study, the force-displacement characteristics can be created for the various values of the input parameters and afterwards they can be linked to the dynamic simulation of the full vehicle system (fvs).

Before the simulation of the fvs within the MBS software, it is recommended to validate created characteristics with the MBS approach. Such a validation can lead to the early detection of the faults within the characteristics. Doing so can lead to the finding of the model, which can be computational faster and might suit better for the dynamic simulation. Also, if the conducted force-displacement characteristics match and their differences are fully understood, the costly experimental verification can be fully replaced by the simulations.

Therefore, the whole fvs dynamic simulation can be verified by the sole experiment, consisting of an accelerometers and a suitable procedure for the data evaluation. After the comparison of the data gathered from the dynamic fvs and the experiment, their correlation coefficient should have a value between the 0,75 and 0,85 .

\section{Acknowledgements}

The presented paper has been prepared with the support of the Ministry of Defence of the Czech Republic, Partial Project for Institutional Development, K-202, Department of Combat and Special Vehicles, University of Defence, Brno.

\section{References}

[1] M. Maloch and S. Cornak, "Study of Prestressed Multileaf Spring Behavior in Terms of Tandem Bogie Layout with Utilization of FEM," in International Conference on Military Technologies (ICMT), Brno, CZ, 2019, vol. 7, pp. 205210.

[2] Yang, Yong, Weiqun Ren, Liping Chen, Ming Jiang, a Yuliang Yang, "Study on ride comfort of tractor with tandem suspension based on multi-body system dynamics," Applied Mathematical Modelling, vol. 33, no. 1 pp. 11-33, 2009.

[3] M. Maloch and S. Cornak, "Mathematical model of the off-road vehicle suspension," in Proceedings of the $22^{\text {nd }}$ International Scientific Conference Transport Means, Trakai, Lithuania, 2018, pp. 495-500.

[4] D. Oatis, "Analyzing Bolt Pretension in the ANSYS Workbench Platform," ANSYS Advantage, vol. 1, no. 4, pp. 2829, 2007.

[5] Richard G. Budynas, J. Keith Nisbett, and Joseph Edward Shigley, "Relating Bolt Torque to Bolt Tension," in Shigley's mechanical engineering design. Tenth edition. New York, NY: McGraw-Hill Education, 2015, pp. 429432. 
[6] R. Lounsbu. (2012, August 12). ANSYS Mechanical Structural Nonlinearities- Lecture 3: Introduction to contact \& Lecture 4: Advanced Contact. [Online]. Available at: http://inside.mines.edu/ apetrell/ENME442/Labs/1301_ENME442_lab6_lecture.pdf

[7] C-J. Kat., "Validated Leaf Spring Suspension Models," Ph.D dissertation, Dept. of Mechanical and Aeronautical Eng, University of Pretoria, Pretoria. 